\title{
Systematically Prioritizing Candidates in Genome-Based Drug Repurposing
}

Anup P. Challa, ${ }^{1}$ Robert R. Lavieri, ${ }^{1}$ Judith T. Lewis, ${ }^{1}$ Nicole M. Zaleski, Jana K. Shirey-Rice, ${ }^{1}$ Paul A. Harris, ${ }^{2}$ David M. Aronoff, ${ }^{3,4}$ and Jill M. Pulley ${ }^{1}$

${ }^{1}$ Vanderbilt Institute for Clinical and Translational Research, Vanderbilt University Medical Center, Nashville, Tennessee. ${ }^{2}$ Department of Biomedical Informatics, Vanderbilt University Medical Center, Nashville, Tennessee.

${ }^{3}$ Division of Infectious Diseases, Department of Medicine, Vanderbilt University School of Medicine, Nashville, Tennessee.

${ }^{4}$ Department of Pathology, Microbiology and Immunology, Vanderbilt University School of Medicine, Nashville, Tennessee.

\section{ABSTRACT}

Drug repurposing is the application of approved drugs to treat diseases separate and distinct from their original indications. Herein, we define the scope of all practical precision drug repurposing using DrugBank, a publicly available database of pharmacological agents, and BioVU, a large, de-identified DNA repository linked to longitudinal electronic health records at Vanderbilt University Medical Center. We present a method of repurposing candidate prioritization through integration of pharmacodynamic and marketing variables from DrugBank with quality control thresholds for genomic data derived from the DNA samples within BioVU. Through the synergy of delineated "target-action pairs," along with target genomics, we identify 230 "pairs" that represent all practical opportunities for genomic drug repurposing. From this analysis, we present a pipeline of 14 repurposing candidates across 7 disease areas that link to our repurposability platform and present high potential for randomized controlled trial startup in upcoming months.

Keywords: drug repurposing, genomics, phenome-wide association study (PheWAS), precision medicine, informatics

\section{INTRODUCTION}

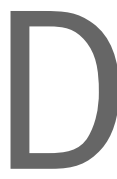

rug repurposing is the practice of developing approved drugs for uses in new, unexplored indications. ${ }^{1}$ Repurposing initiatives have seen major growth in recent years, partially energized by the industrial undertaking of repurposing projects in parallel to the development of new pharmaceuticals. There are numerous benefits of this approach to drug development that are of interest to the pharmaceutical industry. Repurposing is an efficient approach to drug development, as it makes use of existing knowledge on pharmaceuticals and their mechanism(s) of action, thus minimizing the need for much of the preclinical and early safety work required by new chemical entity (NCE) drug discovery and development. Many bioinformaticians believe that drug repurposing is one potential solution to Eroom's Law: the observation that the efficiency of drug development halves about every decade. ${ }^{2}$ Thus, repurposing may offer a systematic approach to increasing the pace of therapeutic development. One of the benefits of drug repurposing is cost reduction; recent estimates suggest that marketing approval through repurposing can cost $\sim 70 \%$ less than that for NCE drug development projects. Drug repurposing is often much faster than NCE drug development: although the time from discovery to regulatory approval of an NCE may require 12 years or longer, repurposing can establish the same task in $<6$ years, nearly half the conventional time. ${ }^{3}$

of recent interest, several large pharmaceutical companies, including Teva Pharmaceutical Industries, Ltd, have sought partnerships with academic bioinformatics centers and data science entities. This has manifested in machine learning partnerships-as with Teva and IBM Watson-through which Teva has made use of Watson's natural language processing abilities to digest unstructured data in electronic health records (EHRs). ${ }^{4}$ Within academia, there is a similar focus on creating industrial relationships, through which academic biobanks partner with pharmaceutical companies to help fuel the development of both repurposable drugs and NCEs. ${ }^{5}$ On the contrary, pharmaceutical partners are eager consumers of research innovations and can provide the industrial machinery required for the reformulation of repurposing candidates, as repurposed drugs can be re-engineered for maximal safety and deliverability before they are included in phase I/II trials. ${ }^{6}$

An issue omnipresent in NCE drug discovery is "off-target" (i.e., of nonintended targets) compound effects, specifically

(c) Anup P. Challa et al. 2019; Published by Mary Ann Liebert, Inc. This Open Access article is distributed under the terms of the Creative Commons License (http:// creativecommons.org/licenses/by/4.0), which permits unrestricted use, distribution, and reproduction in any medium, provided the original work is properly cited. 
when these effects are likely to pose safety concerns or reduce drug efficacy. Although "on-target" toxicity is also a legitimate concern, this issue is more readily addressable, because drug development teams are likely to study the biology of an intended target fully. Hence, "off-target" effects are concerning, as genomic data solely address target biology: genomic data give no information specific to a given drug or molecule's "off-target" effects. With this information, it may be possible to predict the "on-target" toxicity of a molecule of interest-for known target(s)-although genomic data provide no frame of reference for targets that are otherwise unknown. In drug repurposing, however, existing clinical data (about exposure to the drug itself) mitigate the risk of uncovered "off-target" effects: because all potential repurposing candidates have been dosed extensively in humans, it is much less likely-compared with NCE drug discovery-that unexpected "off-target" toxicity issues will emerge in a repurposing project. At present, the failure of most drugs is attributable to lack of efficacy (rather than lack of safety or unacceptable pharmacokinetics); however, it is still true that preclinical safety data are not sufficient to accurately predict the risk of clinical failure from safety issues. ${ }^{7}$ Fortunately, extensive human experience mitigates this safety failure risk for approved drugs, and efficacy failure risk is reduced through the application of human genomic data.

Thus, with the concurrent demands of drug repurposing and personalized medicine, precision drug repurposing is becoming increasingly relevant. The simultaneous analysis of drug databases and genomic data allows for the development of precision repurposing schemes capable of detecting new indications for drugs selected on the basis of human genomic evidence, implicating their targets with specific diseases. Nonetheless, given the large number of available drugs-and the vastly larger space of human genomic information-new methods are required to systematically screen molecules on the pharmacologic and genomic basis of their repurposability.

In this study, we propose an algorithmic scheme for the selection of repurposing candidates using relevant drug profiles from DrugBank, ${ }^{8-12}$ a public database of compounds that have at least been in a phase I clinical trial, and genomic data from BioVU, a biobank of patient samples from Vanderbilt University Medical Center (VUMC). ${ }^{13,14}$ This unbiased, systematic, data-driven approach has allowed us to define the "repurposable drugged genome," stemming from the intersection of the "drugged genome" and targets for which we have high-quality genomic data, as described hereunder.

\section{DrugBank}

DrugBank 5.1.2 (updated December 20, 2018) is a publicly accessible drug referencing tool developed by the University of Alberta; it contains encyclopedia-like entries on common pharmaceutical agents, including $>200$ data fields on each drug entry ("DrugCard"). As a self-described bioinformatics and cheminformatics resource, DrugBank lends itself to multivariate characterization of drug form and function, providing a highly user-friendly application programming interface (API) toward the extraction of relevant data fields. ${ }^{15}$

The most pertinent data sets for this investigation involve DrugBank genomics, described on DrugCards as the associated genes for each drug target. This information facilitates the overlap of drug metrics and BioVU single-nucleotide polymorphism (SNP)/single nucleotide variant (SNV) data through linkage of target genomics; in turn, drug repurposability is evaluable by the minor allele frequency (MAF) and genotype call quality of SNPs/SNVs on drug target genes. This information is then mappable to new indications in the evaluation of phenome-wide association study (PheWAS) data.

For the aforementioned reasons, DrugBank was selected as the source of all "drugged genome" candidates for this investigation. This database was also selected for its regular update schedule (daily DrugCard information uploads, biennial database-wide refreshments ${ }^{9}$ ), breadth of potential repurposing candidates, and flexible API.

\section{BioVU}

BioVU is a repository of DNA samples extracted from excess blood samples in the clinical testing of both adult and pediatric patients at VUMC. The major hallmark of this biobank is its linkage to longitudinal, de-identified EHRs ${ }^{16}$ (Synthetic Derivative $^{17}$ ): samples remain linked to the de-identified medical records of the patients from whom they were collected. The result is a research-ready data set, with patient DNA and genomic data continuously linked to health record information. ${ }^{16}$

In this study, we leverage BioVU to perform PheWAS, ${ }^{18}$ testing for associations between SNPs/SNVs within drug target genes, and clinical phenotypes defined by billing codes. Thus, PheWAS is able to map phenotypes to associated genomic alterations in drug target genes. ${ }^{19,20}$

This study includes the integration of SNP/SNV data from an Illumina Infinium HumanExome BeadChip array (hereafter referred to as "ExomeChip") genotyping platform ${ }^{21}$ in BioVU with the known "drugged genome" from DrugBank. With this synthesis, pharmacodynamic (PD) and genomic attrition algorithms-as defined hereunder and in the Supplementary Data S1 of this article-are applied across both data sets, producing "shortlists" of candidates for drug repurposing. Subsequently, PheWAS call quality for prioritized drug-SNP/ SNV pairs is analyzed as previously described ${ }^{20}$ to support a growing pipeline of drug repurposing projects at VUMC. 


\section{METHODS}

Given there are $>2,000$ approved drugs (inclusive of 3 international regulatory agencies) and $>11,000$ compounds through phase I clinical trials, new methods to improve screening of available compounds are essential to developing new drug repurposing projects. We propose in this report a repurposing prioritization scheme based on candidate targets and mechanisms of action, such that candidates are ranked pragmatically for the launch of new drug repurposing projects.

Thus, the core of this investigation involved our ability to develop an efficient attrition workflow, incorporating VUMC's BioVU/PheWAS data and DrugCards from the mined database of potential candidates.

To extract the pharmacological data necessary for this repurposing project, the complete DrugBank database was downloaded from the repository website (https://www.drug bank.ca/releases/latest). As of November 2017 (update 5.1.0), this database included information on 10,505 drugs, stored in eXtensible Markup Language (XML) format. In the DrugBank XML database, each drug has one record with $>1,700$ descriptive lines.

The following fields were extracted for each drug using the $\mathrm{R}$ programming language ${ }^{22}$ and its XML and plyr packages to scope the entirety of the XML database:

- name

- type ("biotech" or "small molecule")

- status ("approved," "illicit," "investigational," "vet-approved," "nutraceutical," "withdrawn")

- number of targets

- number of targets with known action ("known-action == "yes")

- For each target with known action:

- target name

$\circ$ action

- gene name

- number of enzymes

- list of enzymes

- number of transporters

- list of transporters

Following application of PD and genomic screens relevant to an evaluation of potential repurposing, we extracted data on earliest marketing start date and country of approval for each molecule on a repurposing "shortlist." Country of approval information was scraped for completeness of the approval status data set, and marketing data were extracted as a proxy for potential intellectual property opportunities. Again using the XML package in R, details related to the "products" variable were extracted from the database for each of drug "country" and "started-marketing-on." A custom function in
$\mathrm{R}$, getMarketingDetails, was executed to create a spreadsheet with these marketing details for any desired list of drugs. The $R$ code used to extract relevant information for this drug repurposing study is available in the GitHub repository (https:// github.com/judytlewis/drugRepurposing).

Thus, the entirety of the DrugBank database was first extracted as an XML file using the statistical software R, giving 10,505 potential repurposing candidates $(n=10,505)$. The following variables were then considered:

1. drugName (the listed name of each drug)

2. type (a binary categorization of drug type: small molecule or biologic)

3. status (official categorization by U.S. Food and Drug Administration [FDA] and/or Health Canada (e.g., approved, approved/investigational) $)^{23}$

4. countryApproved (United States and/or Canada) ${ }^{23}$

5. marketingStartDate (date of first marketing, in United States and/or Canada) ${ }^{23}$

6. numberOfTargets (number of known targets for each candidate)

7. numberOfTargetsWithKnownAction (number of known targets with further known pharamacological mechanism for each candidate)

8. target (i) (listed target for each candidate, with $n$ representing iteration per known target in numberOfTargets $(i \in[1,25]))$

9. action (j) (listed action (e.g., inhibitor, activator) for each candidate, with $j$ representing iteration per known target in numberOfTargets $(j \in[1,25]))$

10. geneName $(k)$ (associated gene for each target in numberOfTargets, iterated $k$ times $(k \in[1,25]))$

Controlled analysis of each of these parameters allowed for efficient attrition, by which systematic consideration of PD for each candidate was used as a basis for stepwise parsing. Thus, the following screens were applied, given the task of defining the "repurposable drugged genome."

\section{Drugs by Type}

Given the significant cost difference of obtaining small molecules for clinical trials, as compared with biologics, ${ }^{24}$ and the binary nature of drug type classification, ${ }^{24}$ separation of mined agents as "biologics" and "small molecules" quickly identified drugs more easily accessible (i.e., small molecules) from those generally less accessible (i.e., biologics). Our list is intended to be both computationally valid and pragmatic in its application to high-throughput screening of identified drug repurposing candidates. Therefore, biologics were excluded after this first round of analysis, given the practical difficulties 
in obtaining many of these agents. ${ }^{25-27}$ This discrepancy between small molecules and biologics is easily illustrated by comparing the small molecule misoprostol ${ }^{28,29}$ to biologics that would also make sense to repurpose for a similar new use.

Namely, misoprostol is a prostaglandin-derived small molecule currently approved for the treatment of iatrogenic ulcers, resulting from overuse of nonsteroidal anti-inflammatory drugs (NSAIDs). ${ }^{28}$ An ongoing randomized, double-blind, placebo-controlled, phase II clinical trial (NCT03617172) led by Dr. David Aronoff from VUMC is testing the repurposability of misoprostol for the prevention of recurrent Clostridioides difficile infection, the leading cause of antibiotic-associated diarrhea. ${ }^{28}$ Per generalized DrugBank market data, the median price per tablet of misoprostol is $\$ 2.33 .{ }^{30}$ In contrast, the cost of obtaining a biologic agent similarly indicated for gastritis (e.g., adalimumab $^{26}$ ) prohibits purchasing large amounts of the biologic necessary to conduct a clinical trial. A review of market data for adalimumab gives the average cost per dose in the United States to be $\$ 2,669,{ }^{31}$ roughly 1000 -fold greater than the average price per tablet of misprostol. Similarly, a recent study estimates the price of bezlotoxumab, a biologic agent for preventing recurrent $C$. difficile infection, as $\$ 4,560$ per vial. ${ }^{32}$ Given that biologics are often proprietary, although many established small molecules are off-patent, the issue of limited drug access plagues repurposing studies of biologics. Removing all biologics left 9,292 potential repurposing candidates available for review from the total listing of 10,505 drugs.

\section{Drugs by Approval Status}

A major aim of repurposing is the development of new therapeutic strategies among sets of agents currently in use for a wide variety of indications. For repurposing to remain practical within the academic medical center setting and within a reasonable timeframe, repurposing candidates must be approved for clinical use, or at least through a phase I clinical trial.

The data in DrugBank include approval status for each cataloged agent in keeping with the labels established by the FDA, European Medicines Agency, and Health Canada. ${ }^{23}$ Therefore, only drugs with a listing "Approved" were considered as repurposing candidates. Specifically, drugs with the labels provided in Appendix Table A1 were retained for further consideration.

Attrition by approval status left 2,219 potential repurposing candidates from the total listing of 10,505 investigational drugs.

\section{Drugs by Number of Targets of Known Mechanisms of Action}

Noting the focus of drug repurposing methods on "selective" drugs (ideally, drugs of one target of known mechanism of action [MOA]), we decided to consider only drugs with one known target and MOA. Although future studies may consider more complex pharmacology (i.e., drugs with multiple known targets [and thereby multiple gene targets for analysis]), the preliminary nature of this scan dictated restriction to drugs of one target with known MOA.

Parsing by targets of known MOA left 823 potential repurposing candidates from the original listing of 10,505 investigational drugs.

Exclusions. Based on existing knowledge of drug toxicity, drugs of limited potential owing to significant safety issues were manually removed from the agent shortlist, in consultation with pharmacologists. Our repurposing method relies on human genomic data ${ }^{20}$; thus, we focus solely on drugs with human protein targets. Hence, drugs with nonhuman targets (fungal, viral, helminthic, and bacterial nucleic acids) were removed from this data set. Given that many anticancer agents work by damaging DNA (e.g., alkylating agents), these drugs were also excluded from consideration. Drug entries with missing or null entries in any aforementioned data field were additionally parsed.

Consolidations. Given that drugs of the same class (e.g., ACE inhibitors) are inherently redundant in affecting the same target, we decided to move from a per-drug attrition scheme to a per-drug class parsing strategy. To accomplish this task, "target-action pairs" were defined through the simultaneous association of each drug target and its DrugBank-specified action (e.g., inhibitor, activator). Thus, all drugs were grouped into "target-action pairs" and handled in this manner for the remainder of the study.

After exclusion and consolidations, 621 remaining small molecules were grouped into 237 unique "target-action pairs." MAF data-a cutoff of $0.1 \%$ generally means we have sufficient data to run the PheWAS analysis and observe meaningful/new associations for a given drug target-were then integrated into the list of agents to reach a working, precision model.

\section{Merger with Genomic Data}

First, a comprehensive list of SNPs/SNVs on the exome chip $^{21}$ (see BioVU section)-along with their unique ExomeChip ID numbers (exmID), reference cluster ID numbers $(\mathrm{rsID}),{ }^{33}$ gene name (Gene), MAF, mutation type and listing (Mutation), major base pair (A1), and minor base pair (A2)were mined from the Chip gene annotation file and BioVU databank of genotypes to an XML file using the statistical software R. This gave 239,796 SNPs/SNVs available for review. 
A selectivity screen was then applied to the mined SNP/SNV data: first, SNPs/SNVs with rsID listed as "NULL" were removed from consideration, given the inability to verify SNP/ SNV information with established genomics databases, including dbSNP (https://www.ncbi.nlm.nih.gov/snp). ${ }^{33}$ For consistency in data handling, we considered only SNP/SNV querying by rsID; we acknowledge that this method does not allow for enrichment of our SNP/SNV data set outside dbSNP, by neglecting the possibility of discovering additional SNP/ SNV information from another database. However, given that dbSNP information that we do not curate is most likely dominated by rare variants ${ }^{34}$-and our purging of rare variants from consideration, for practical reasons-we do not consider this limitation to be significant.

Next, SNPs/SNVs with genotyping missingness $>0.05$ and MAF $<0.001$ for white populations-the dominant demographic represented in the available PheWAS data-were removed from the ExomeChip. Given the implementation of PheWAS-based algorithms in this investigation, $0.1 \%$ frequency was used as "utility" benchmark, noting optimization of PheWAS performance at MAF $>0.001$ and establishment of this limit as an appropriate cutoff in previous literature. ${ }^{35,36}$ Indeed, using frequency conventions used by the National Center for Biotechnology Information, ${ }^{35}$ MAF values $\leq 0.001$ are deemed "rare," rather than "minor."

Under an assumption of stochastic missingness, ${ }^{37}$ SNPs/ SNVs with missingness $>5 \%$ were parsed from the model to prevent confounding bias within phenotypic associations. ${ }^{38}$

Application of these screens gave 58,945 eligible SNPs/ SNVs-tied to (de-identified) patient electronic medical records at VUMC-from the original listing of 237,796 variants.

Synthesis of the pharmacological data and narrowed ExomeChip data were now feasible, whereby shortlisted drugs were further reduced on coverage of target genes in our genomic data set. This was accomplished by application of the "target-action pairs" strategy, allowing genomic comparison between target-associated genes and eligible SNPs/SNVs. The number of "target-action pairs" with genes and eligible SNPs/ SNVs cross-listed on our ExomeChip was then determined. Thus, it was determined that 227 "target-action pairs" of the pool of 237 "pairs" demonstrated cross-listed SNPs/SNVs, giving 96\% total SNP/SNV coverage for the ExomeChip population in BioVU. These 227 distinct "target-action pairs" translate to 147 unique targets that may be further probed for repurposing potential.

A representation of the holistic attrition strategy is given in Appendix Figure A1. Example "target-action" pairings are listed in Appendix Table A2. Furthermore, Supplementary Data S2 to this article is given, which may be accessed as
"Supplement.md" through the GitHub repository at https:// github.com/judytlewis/drugRepurposing. In this supplement, we provide listings of drugs and "target-action pairs" considered at each stage of the above-described filtration, and a listing of the 227 "target-action pairs" we believe to encompass all pragmatic opportunities in genome-based drug repurposing and their associated marketing information.

\section{DISCUSSION OF RESULTS AND STUDY LIMITATIONS}

Our model effectively produces a prioritized set of drugs that are promising candidates for our specific method of repurposing, by reducing $\sim 11,000$ drugs and $\sim 240,000$ SNPs/ SNVs to a prioritized set of 227 "target-action pairs," given specific criteria on drug target number and approval status, along with SNP/SNV coverage and MAF.

Nonetheless, two conditions restrict our outputs, in that this model only operates on small molecules with one target of known MOA. Given that 518 agents of the 621 drugs with one target of known MOA have only one total target listed in DrugBank (i.e., 83.41\% one-target-total rate), the aforementioned statement may be simplified to a requirement of small molecules of no more than one total target. Clearly, drug specificity is a relative concept and a complete specificity/ activity profile against all possible drug targets does not exist for any drug, much less all approved drugs. However, we have taken some obvious quality control steps, such as removing DNA damaging agents and drugs known to affect nonhuman targets from our data set.

The above assumptions were used both in consideration of the ideal characteristics for repurposing candidates, along with an understanding of this investigation as a preliminary attempt at the design of a repurposing candidate "search engine." In short, this investigation aimed to develop a method by which the entirety of the "drugged genome" may be further narrowed to the "repurposable drugged genome."

In addition, drug toxicity was not considered systematically in the attrition of potential repurposing candidates. Although candidates with severe and obvious toxicity concerns-as relevant to utility for repurposing-were manually removed, it was not feasible to mine structured toxicology data from DrugBank, given the absence of an absolute measure of toxicity in the field of pharmacology. In addition, safety issues were difficult to assess, as these remain relative to selected patient populations and besought indications.

On the whole, our method of repurposability screening produces a shortlist of 147 unique targets that may be considered further for drug repurposing. This result is derived through PD screening of all approved small molecules across 
established governmental regulatory organizations, along with genomic screening of all variants associated with their druggable targets. Hence, our filtration reflects pharmacological, genomic, and pragmatic considerations of drug repurposing. Indeed, one cannot understate the importance of real-world considerations in the selection of drug repurposing candidates. Even for a large, relatively well-funded drug repurposing program-such as that at VUMC-time and money are limiting factors, as the identification and phase II efficacy study of a repurposing candidate often requires $\$ 3-\$ 8$ million and several years of time. Therefore, it is only feasible for an academic medical center-based program to launch a handful (or fewer) of repurposing programs in a given year.

Our method of repurposability screening accommodates these limitations by providing a comprehensive database of all potential leads for genomic drug repurposing. This framework supports the upstart of new randomized controlled trials (RCTs), as all candidates for which genomic drug repurposing is possible are listed in a centralized location. In turn, repurposing programs using this resource may save significant drug discovery resources necessary for scoping the existing pharmacopeia for repurposing hits.

Our repurposing program has used a similar strategy in the generation of its repurposing pipeline. Thus, we present test cases of the above workflow across seven disease areas, for which we pull genomic repurposing shortlists from the output of systematic pharmacological and genomic analyses. Hence, our current pipeline of 14 repurposing candidates (including 3 drugs in ongoing RCTs [NCT03694249, NCT03617172, NCT03527472]) is derived from workflows analogous to those in this article; because all these molecules are in funded clinical trials, we conclude that the attrition method presented above is largely successful in supporting trial startup.

A summary of our current drug repurposing pipeline-as traceable to this study-is given in Appendix Table A3.

We also compare our findings with those presented in a recent scope of the genomic repurposing space by Finan et $a l .{ }^{39} \mathrm{In}$ this publication, Finan et al. ${ }^{39}$ present genome-wide association study (GWAS)-derived target-SNP pathogenicity relationships that have application in stimulating new drug repurposing projects. The authors assert that the druggable genome-defined by 4,479 genes corresponding to protein targets able to bind available large and/or small molecules $^{39,40}$-may be reduced to a total of 144 drug repurposing targets, as identified by analysis of SNP pathogenicity in druggable genes within their GWAS data sets. ${ }^{39}$ Our analysis presents a similar statistic while focusing on the identification of repurposing candidates and considering drug-specific attrition criteria and target directionality. Thus, we count 147 unique targets that are eligible candidates for repurposing. Our methods contain pharmacological screening of potential repurposing candidates, identifying repurposable agents in addition to their intended targets. Although their study is the closest probe to ours available in the literature, Finan et al. ${ }^{39}$ do not fully link shortlisted targets to drug candidates, as they do not screen targets by the PD of their associated agents. The authors also do not consider the pragmatic considerations necessary for a drug repurposing project ${ }^{39}$; we address these concerns by considering only "Approved" small molecules, which are ideal candidates for repurposing. Finan et al. also state that target agonists and antagonists present differences in repurposablity; however, they do not apply this reasoning to further prioritize their target shortlist. ${ }^{39}$ We acknowledge that repurposablity is a function of target MOA by creating "target-action pairs," shifting our analysis of repurposability away from target information alone and focusing more on the pharmacology of available agents that could be repurposed.

The comparison of our results with those of Finan et al. ${ }^{39}$ is given in Appendix Figure A2. We note that our procedure of removing "target-action pairs" with obvious and severe toxicity concerns is similar to the toxicity attrition scheme used in Finan et al. ${ }^{39}$

By comparing our approach to Finan et al., ${ }^{39}$ we define the "repurposable drugged genome": a new scope for the genomic repurposing space, consisting of 227 "target-action pairs." We note that 147 targets (Appendix Fig. A2) within the druggable genome are associated with a repurposable, marketed small molecule. However, a large majority of targets within the druggable genome have not been harnessed for their de novo drug development potential. ${ }^{41}$ As new molecules for these targets are developed-and genomic association studies reveal significant SNVs within their associated genes-our definition of the scope of genomic drug repurposing will surely change.

\section{CONCLUSIONS AND RECOMMENDATIONS FOR FUTURE STUDY}

Drug repurposing, as we approach it, is an interdisciplinary endeavor, benefitting from the integration of biomedical informatics, pharmacology, and genomics. We are most interested in using this approach to develop therapies for diseases with no available, effective treatments-regardless of the incidence of the disease or commercial potential. Our drug repurposing program, at present, has 3 clinical trials in progress (NCT03694249, NCT03617172, NCT03527472), included in the 14 total projects spread across 7 disease areas that we currently seek to address. Given that we establish the scope of all genomic drug repurposing, we observe that the workflow presented in this article is effective in reducing the necessary 
investment of time and money for lead identification in a drug repurposing RCT.

Indeed, the progress of our group toward NCT03617172 ("PROCLAIM-Prevent Recurrence of Clostridium difficile Infection with Misoprostol") highlights the power of our approach to identify strong precision drug repurposing hits. This phase II RCT seeks to assess the safety and efficacy of misoprostol-originally indicated for the treatment of NSAID-induced ulceration and postpartum hemorrhage-in the prevention of recurrence of $C$. difficile infection in patients of at least 18 years of age during the first 8 weeks after completion of standard of care oral antibiotic therapy. A repurposing signal between misoprostol and an SNP on the type 2 prostaglandin E receptor (PTGER2) was detected by a workflow similar to that in this article; the associated PTGER2 PheWAS hits were significantly enriched for gastritis and duodenitis, and esophageal ulcer. ${ }^{42}$ More information on the design of NCT03617172 is available in Appendix Figure A3.

Overall, we propose an efficient data filtering and integration model, considering pharmacological data from a publicly available database and genomic data in a large-scale DNA repository. This allows for the entirety of the "drugged genome" to be reduced to a prioritized set of drugs that may be further considered for repurposing potential.

Future enhancement of this model will address the aforementioned limitations in drug screening. Enhanced consideration of a systematic attrition strategy for drug toxicity would be useful, requiring first the identification of an easily mineable variable available at a centralized location. This investigation provides a functional definition of repurposing within the "drugged genome." Future work utilizing this study as a tool may explore PheWAS signals of shortlisted drugs generated from increasingly specified search criteria. Thus, this study provides a framework upon which later investigations can rely in determination of optimal repurposing candidates across the "drugged genome."

We intend to use this framework in the continuing selection of drug repurposing candidates for our pipeline.

\section{ACKNOWLEDGMENT}

The authors acknowledge the assistance of Helen Naylor, Assistant Director for Precision Medicine, Vanderbilt University Medical Center, in the completion of this study.

\section{AUTHORS' CONTRIBUTIONS}

A.P.C. developed the attrition filters for mined pharmacological and genomic data, executed "target-action pairing," and drafted this article. R.R.L. worked with A.P.C. to design pharmacodynamic, pharmacoeconomic, and genomic data filters, draft this article, and revise the article. J.T.L. developed programs to extract relevant pharmacological information from DrugBank. N.M.Z. designed and created the figures in this article. J.K.S.R. revised this article for technical accuracy and provided statistics on our drug repurposing program. P.A.H. revised this article. D.M.A. revised this article and provided information on the progress of RCTs under our repurposing program. J.M.P. designed figures with N.M.Z. and revised this article.

\section{DISCLOSURE STATEMENT}

The authors declare no competing interests.

\section{FUNDING INFORMATION}

Research reported in this publication was supported by the National Center for Advancing Translational Sciences of the National Institutes of Health under Award Number U54TR02243-02. The content is solely the responsibility of the authors and does not necessarily represent the official views of the National Institutes of Health.

\section{SUPPLEMENTARY MATERIAL}

Supplementary Data S1

Supplementary Data S2

\section{REFERENCES}

1. Corsello SM, Bittker JA, Liu Z, et al:: The Drug Repurposing Hub: a nextgeneration drug library and information resource. Nat Med 2017;23:405-408.

2. Nosengo N: Can you teach old drugs new tricks? Nature 2016;534:314-316.

3. Scannell JW, Blanckley A, Boldon $H$, Warrington B: Diagnosing the decline in pharmaceutical R\&D efficiency. Nat Rev Drug Discov 2012;11:191-200.

4. Teva Pharmaceuticals and IBM Expand Global Partnership to Enable Drug Development and Chronic Disease Management with Watson. www.tevapharm .com/news/teva_pharmaceuticals_and_ibm_expand_global_partnership_to_ enable_drug_development_and_chronic_disease_management_with_watson_ 10_16.aspx (Last accessed on April 13, 2018).

5. Bayer, Broad Expand Partnership to Advance Cancer Drug Discovery Research. Broad Institute (2017). https://www.broadinstitute.org/news/bayer-broadexpand-partnership-advance-cancer-drug-discovery-research (Last accessed on April 17, 2018).

6. Murteira S, Ghezaiel Z, Karray S, Lamure M: Drug reformulations and repositioning in pharmaceutical industry and its impact on market access: reassessment of nomenclature. J Mark Access Health Policy 2013;1.

7. Cook D, Brown D, Alexander $R$, et al:: Lessons learned from the fate of AstraZeneca's drug pipeline: a five-dimensional framework. Nat Rev Drug Discov 2014;13:419-431.

8. Wishart DS, Feunang YD, Guo AC, et al.: DrugBank 5.0: a major update to the DrugBank database for 2018. Nucleic Acids Res 2018;46:D1074-D1082.

9. Law V, Knox C, Djoumbou Y, et al.: DrugBank 4.0: shedding new light on drug metabolism. Nucleic Acids Res 2014;42:D1091-D1097.

10. DrugBank 3.0: a comprehensive resource for 'Omics' research on drugs | Nucleic Acids Research | Oxford Academic. https://academic.oup.com/nar/article/39/ suppl_1/D1035/2507041 (Last accessed on April 17, 2018). 
11. Wishart DS, Knox C, Guo AC, et al:: DrugBank: a knowledgebase for drugs, drug actions and drug targets. Nucleic Acids Res 2008;36:D901-D906.

12. Wishart DS, Knox C, Guo AC, et al:: DrugBank: a comprehensive resource for in silico drug discovery and exploration. Nucleic Acids Res 2006;34:D668-D672.

13. Danciu I, Cowan JD, Basford $M$, et al.: Secondary use of clinical data: the Vanderbilt approach. J Biomed Inform 2014;52:28-35.

14. McGregor TL, Van Driest SL, Brothers KB, et al.: Inclusion of pediatric samples in an opt-out biorepository linking DNA to de-identified medical records: pediatric BioVU. Clin Pharmacol Ther 2013;93:204-211.

15. About DrugBank-DrugBank. https://www.drugbank.ca/about (Last accessed on April 10, 2018)

16. Roden DM, Pulley JM, Basford MA, et al:: Development of a large-scale deidentified DNA biobank to enable personalized medicine. Clin Pharmacol Ther 2008;84:362-369.

17. Synthetic Derivative | Department of Biomedical Informatics. https://www. vumc.org/dbmi/synthetic-derivative (Last accessed on April 19, 2018).

18. Wei W-Q, Bastarache LA, Carroll RJ, et al:: Evaluating phecodes, clinical classification software, and ICD-9-CM codes for phenome-wide association studies in the electronic health record. PLoS One 2017;12:e0175508.

19. Denny JC, Bastarache LA, Ritchie MD, et al.: Systematic comparison of phenomewide association study of electronic medical record data and genome-wide association study data. Nat Biotechnol 2013;31:1102-1111.

20. Pulley JM, Rhoads JP, Jerome RN, et al:: Accelerating precision drug development and drug repurposing by leveraging human genetics. ASSAY Drug Dev Technol 2017;15:113-119.

21. Guo Y, He J, Zhao S, et al.: Illumina human exome genotyping array clustering and quality control. Nat Protocols 2014;9:2643-2662.

22. R: The R Project for Statistical Computing. https://www.r-project.org (Last accessed on May 15, 2018).

23. Documentation and Sources-DrugBank. https://www.drugbank.ca/documentation (Last accessed on April 27, 2018).

24. Pharmaceuticals | Pharmaceuticals | Bayer-Small and large molecules http:|| pharma.bayer.com/en/innovation-partnering/technologies-and-trends/small-andlarge-molecules (Last accessed on May 10, 2018).

25. The Conversation. Biologics: The Pricey Drugs Transforming Medicine. Scientific American. https://www.scientificamerican.com/article/biologics-the-pricey-drugstransforming-medicine (Last accessed on November 2, 2018).

26. Adalimumab. https://www.drugbank.ca/drugs/DB00051 (Last accessed on November 2, 2018).

27. Psoriasis Treatment Cost Comparison: Biologics Versus Home Phototherapy https://www.ajpb.com/journals/ajpb/2018/ajpb_januaryfebruary2018/psoriasistreatment-cost-comparison-biologics-versus-home-phototherapy (Last accessed on November 2, 2018).

28. Project Information-NIH RePORTER-NIH Research Portfolio Online Reporting Tools Expenditures and Results. https://projectreporter.nih.gov/project_info_ description.cfm?aid=9205096 \&ticde $=32303027 \&$ tddparam $=\varepsilon$ tddvalue $=\varepsilon t d d s u b=$ $\varepsilon t c r=1 \&$ csb=defaultetcs=ASCEtpball=(Last accessed on November 8, 2018)

29. NCATS issued 11 Bench-to-Clinic awards. National Center for Advancing Translational Sciences. 2016. https://ncats.nih.gov/ntu/projects/2016 (Last accessed on November 8, 2018).

30. Misoprostol-DrugBank. https://www.drugbank.ca/drugs/DB00929 (Last accessed on November 9, 2018).

31. Average price of Humira by country 2015 | Statistic. Statista. https://www .statista.com/statistics/312014/average-price-of-humira-by-country (Last accessed on November 9, 2018).

32. Prabhu VS, Dubberke ER, Dorr MB, et al:: Cost-effectiveness of bezlotoxumab compared with placebo for the prevention of recurrent Clostridium difficile infection. Clin Infect Dis 2018;66:355-362.

33. dbSNP Home Page. https://www.ncbi.n/m.nih.gov/projects/SNP (Last accessed on May 14, 2018).
34. Rare and Common Variants: Twenty arguments. https://www.ncbi.nlm.nih.gov/ pmc/articles/PMC4408201 (Last accessed on May 31, 2019).

35. Human Variation Sets in VCF Format. https://www.ncbi.nlm.nih.gov/variation/ docs/human_variation_vcf (Last accessed on May 19, 2018).

36. Sulovari A, Li D. GACT: a Genome build and Allele definition Conversion Tool for SNP imputation and meta-analysis in genetic association studies. BMC Genomics 2014;15:610.

37. Lin W-Y, Liu N: Reducing bias of allele frequency estimates by modeling SNP genotype data with informative missingness. Front Genet 2012;3:107.

38. James I, McKinnon E, Gaudieri S, Morahan G; Diabetes Genetics Consortium: Missingness in the T1DGC MHC fine-mapping SNP data: association with HLA genotype and potential influence on genetic association studies. Diabetes Obes Metab 2009;11(Suppl. 1):101-107.

39. Finan C, Gaulton A, Kruger FA, et al:: The druggable genome and support for target identification and validation in drug development. Sci Trans/ Med 2017; 9:eaag 1166.

40. The druggable genome | Nature Reviews Drug Discovery. https://www.nature .com/articles/nrd892 (Last accessed on January 1, 2019).

41. Unexplored therapeutic opportunities in the human genome / Nature Reviews Drug Discovery. https://www.nature.com/articles/nrd.2018.14 (Last accessed on June 13, 2019).

42. PROCLAIM-Misoprostol in the prevention of recurrent CDI prevent recurrence of Clostridium difficile infection with misoprostol-full text view-Clinical Trials.gov. https://clinicaltrials.gov/ct2/show/NCT03617172 (Last accessed on November 9, 2018).

Address correspondence to: Anup P. Challa

Vanderbilt Institute for Clinical and Translational Research Vanderbilt University Medical Center 2525 West End Avenue, Suite 600 Nashville, TN 38163

E-mail: anup.p.challa.1@vumc.org

\section{Abbreviations Used}

$$
\begin{aligned}
\text { ACE } & =\text { angiotensin-converting enzyme } \\
A P I & =\text { application programming interface } \\
E H R & =\text { electronic health record } \\
\text { exmID } & =\text { ExomeChip ID number } \\
\text { FDA } & =\text { U.S. Food and Drug Administration } \\
\text { GWAS } & =\text { genome-wide association study } \\
\text { MAF } & =\text { minor allele frequency } \\
\text { MOA } & =\text { mechanism of action } \\
\text { NCE } & =\text { new chemical entity } \\
\text { NSAID } & =\text { nonsteroidal anti-inflammatory drug } \\
\mathrm{PD} & =\text { pharmacodynamic } \\
\text { PheWAS } & =\text { phenome-wide association study } \\
\text { RCT } & =\text { randomized controlled trial } \\
\mathrm{rSID} & =\text { reference cluster ID number } \\
\mathrm{SNP} & =\text { single-nucleotide polymorphism } \\
\mathrm{SNV} & =\text { single nucleotide variant } \\
\mathrm{SOC} & =\text { standard of care } \\
\mathrm{VUMC} & =\text { Vanderbilt University Medical Center } \\
\mathrm{XML} & =\text { eXtensible Markup Language }
\end{aligned}
$$




\section{Appendix}

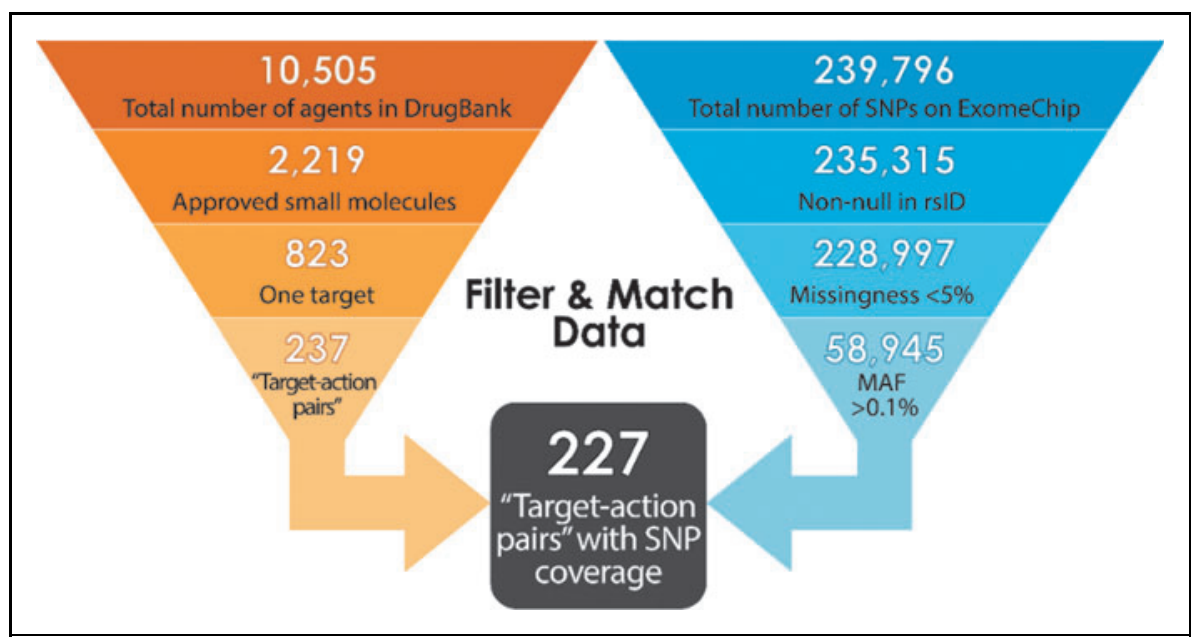

Appendix Fig. A1. A summary representation of the pharmacodynamic and genomics-inspired workflow developed for the selection of optimal repurposing candidates: attrition at each step is defined through genomic and chemical profiling of ideal small molecule candidates. MAF, minor allele frequency; rsID, reference cluster ID number; SNP, single-nucleotide polymorphism.

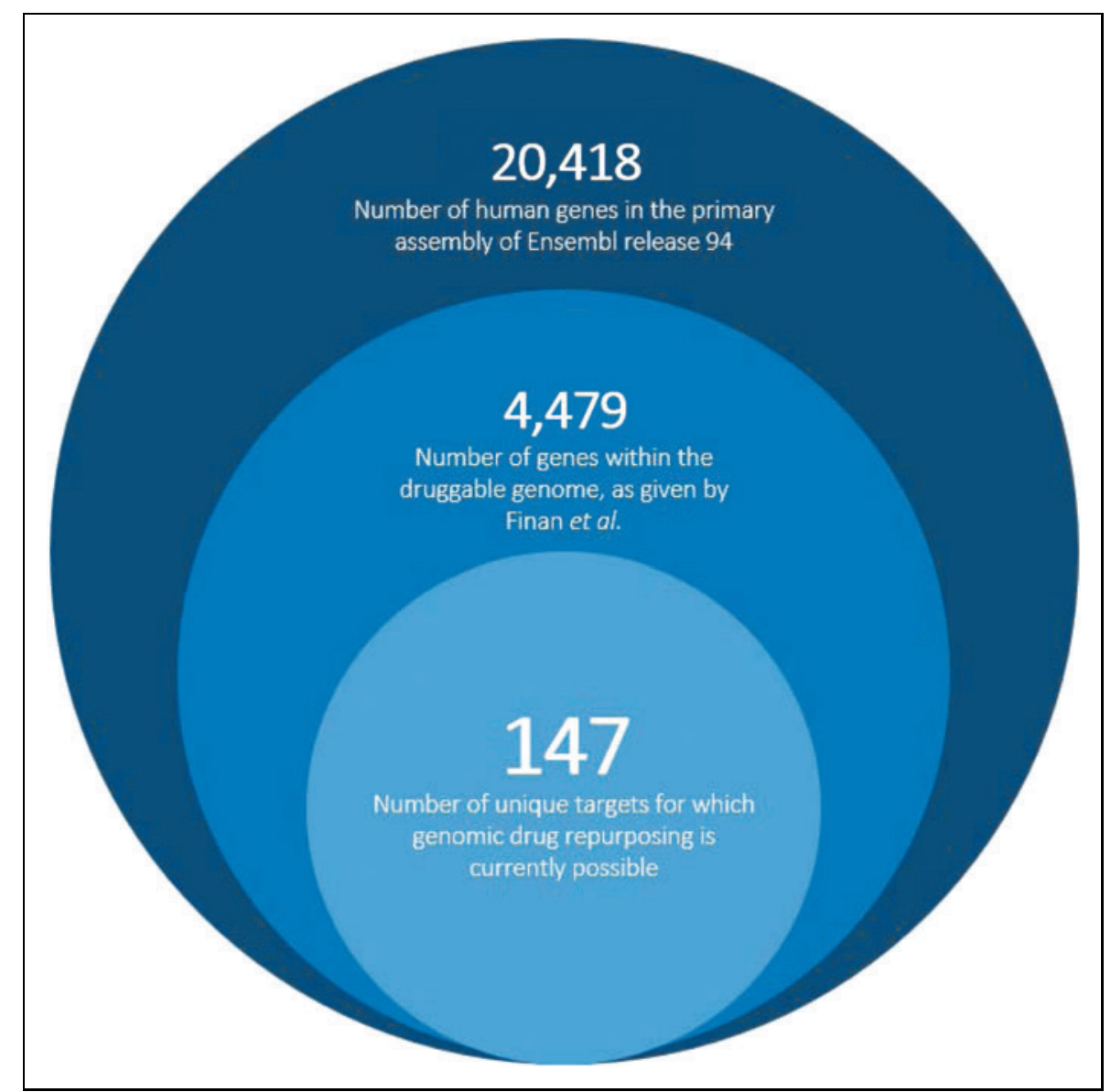

Appendix Fig. A2. Our repurposability screens define the current scope of the genomic drug repurposing space (227 "target-action pairs" of 147 unique targets) within the entirety of the druggable genome. ${ }^{\mathrm{A} 1, \mathrm{~A} 2}$ 
Confirm CDI resolution, enroll, randomize, and monitor for symptoms.

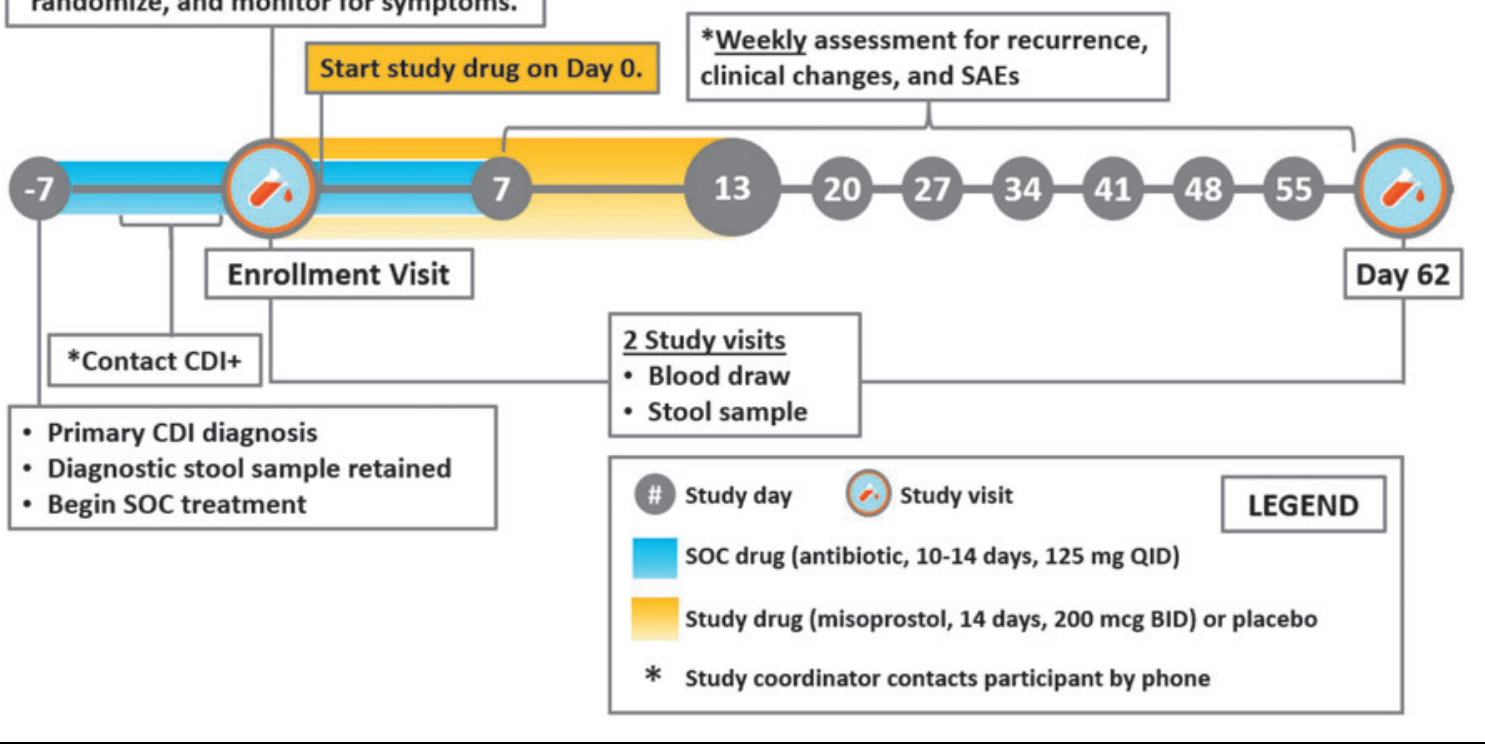

Appendix Fig. A3. A summary of the trial design of NCT03617172, a phase II repurposing randomized controlled trial for the potency and efficacy of misoprostol to treat recurrent Clostridioides difficile infection. ${ }^{\mathrm{A} 3} \mathrm{BID}$, twice per day; CDI, $C$. difficile infection; QID, four times per day; SAE, serious adverse event; SOC, standard of care.

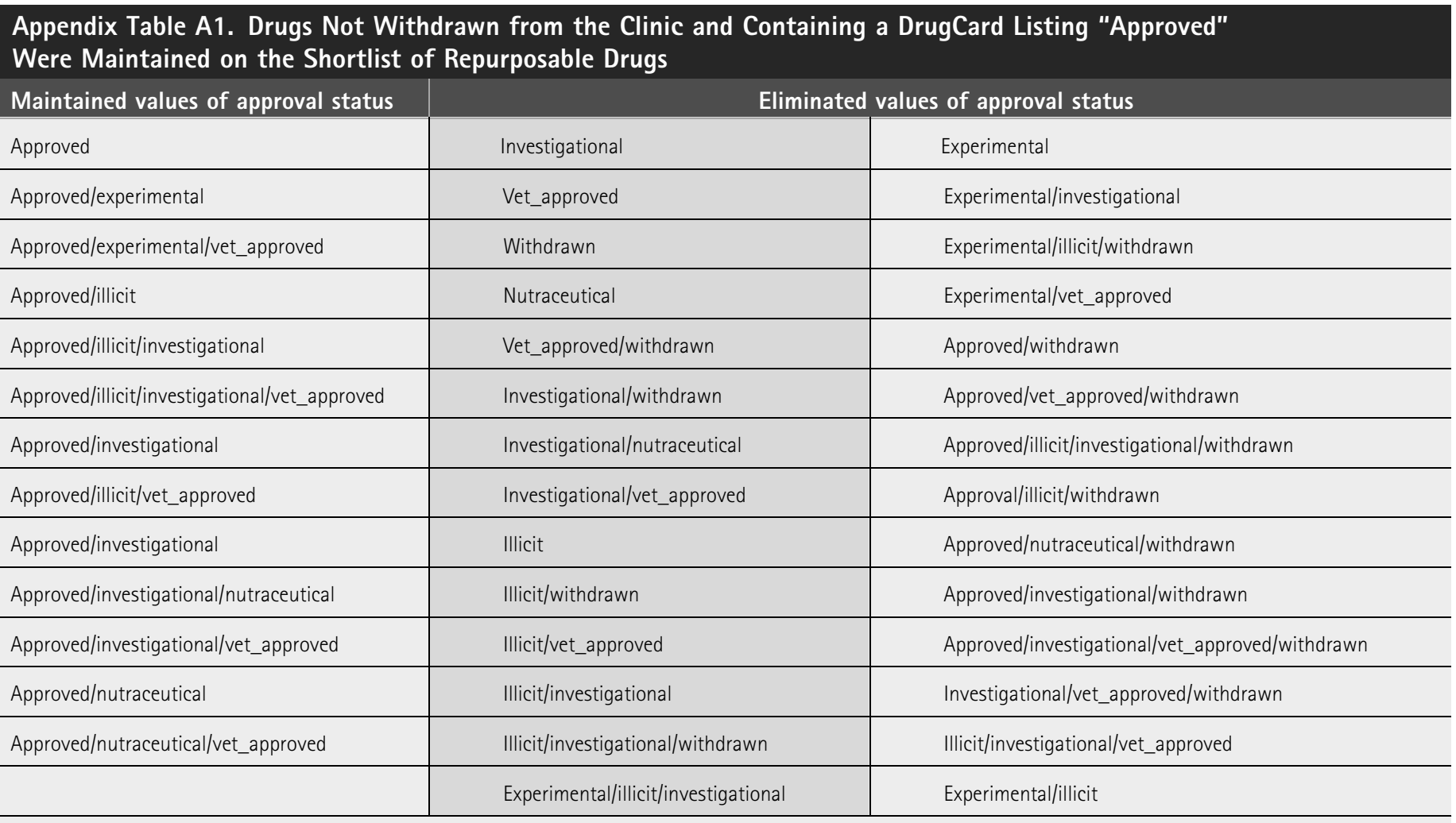

In contrast, drugs without this keyword-or those given as "Withdrawn" and "Approved"-were removed from consideration as nonrepurposable small molecules. 
Appendix Table A2. A Selection of "Target-Action Pairs," Each with MAF_W $>0.001$ and $<5 \%$ Genotype Missingness for at Least One Single-Nucleotide Polymorphism/Single Nucleotide Variant from the ExomeChip

\section{Target}

Mechanism of action Small molecule

Muscarinic acetylcholine receptor $\mathrm{M} 1$
Current indication(s)

Marketing start date

\begin{tabular}{|c|c|c|c|c|}
\hline & Antagonist & Cyclopentolate & Mydriasis and cycloplegia (for diagnostic purposes) & June 30, 1958 \\
\hline & Antagonist & Glycopyrronium & $\begin{array}{l}\text { Salivary, tracheobronchial, and pharyngeal secretions, } \\
\text { acid reflux, cardiac vagal inhibitory reflexes during induction } \\
\text { of anesthesia and intubation, COPD }\end{array}$ & March 28, 1961 \\
\hline & Antagonist & Scopolamine & $\begin{array}{l}\text { Colicky abdominal pain, bradycardia, sialorrhea, diverticulitis, } \\
\text { IBS, motion sickness }\end{array}$ & January 1, 1966 \\
\hline & Antagonist & Clidinium & Peptic ulcer disease, colicky abdominal pain, diverticulitis, IBS & September 1, 1966 \\
\hline & Antagonist & Propantheline & Enuresis, hyperhidrosis, abdominal spasm, bladder spasm & December 14, 1981 \\
\hline & Antagonist & Pirenzepine & Peptic ulcer, gastric ulcer, and duodenal ulcer. & December 31, 1984 \\
\hline & Antagonist & Trihexyphenidyl & Parkinson's disease, extrapyramidal reactions & November 1, 1987 \\
\hline \multirow{2}{*}{$\begin{array}{l}\text { Tyrosine-protein } \\
\text { kinase BTK }\end{array}$} & Inhibitor & Acalabrutinib & Mantle cell lymphoma & October 31, 2017 \\
\hline & Inhibitor & Ibrutinib & $\begin{array}{l}\text { Mantle cell lymphoma, chronic lymphocytic leukemia, } \\
\text { Waldenstrom's macroglobulinemia }\end{array}$ & November 7, 2013 \\
\hline \multirow{4}{*}{$\begin{array}{l}\text { Prostaglandin F2-alpha } \\
\text { receptor }\end{array}$} & Agonist & Tafluprost & \multirow[t]{3}{*}{ Elevated intraocular pressure } & February 10, 2012 \\
\hline & Agonist & Travoprost & & October 20, 2006 \\
\hline & Agonist & Latanoprost & & March 20, 1995 \\
\hline & Agonist & $\begin{array}{l}\text { Dinoprost } \\
\text { tromethamine }\end{array}$ & $\begin{array}{l}\text { Abortion of second-trimester pregnancy, induction } \\
\text { of labor, vasodilation (for diagnostic purposes) }\end{array}$ & December 31, 1974 \\
\hline \multirow{3}{*}{$\begin{array}{l}\text { Alpha- } 1 \text { A adrenergic } \\
\text { receptor }\end{array}$} & Agonist & Tetryzoline & Minor eye irritation & November 30, 1979 \\
\hline & Agonist & Methoxamine & Hypotension & December 31, 1950 \\
\hline & Agonist & Ergonovine & Postpartum hemorrhage, postabortion hemorrhage & December 31, 1939 \\
\hline \multirow{2}{*}{$\begin{array}{l}\text { Alpha- } 1 \text { A adrenergic } \\
\text { receptor }\end{array}$} & Antagonist & Silodosin & \multirow[t]{2}{*}{$\mathrm{BPH}$} & March 23, 2009 \\
\hline & Antagonist & Tamsulosin & & September 12, 1997 \\
\hline $\begin{array}{l}\text { Heat-stable } \\
\text { enterotoxin receptor }\end{array}$ & Agonist & Linaclotide & IBS with constipation, chronic idiopathic constipation & August 30, 2012 \\
\hline Toll-like receptor 7 & Agonist & Imiquimod & Facial actinic keratosis, genital and perianal warts & February 25, 2010 \\
\hline \multirow[t]{3}{*}{ P2Y purinoceptor 12} & Antagonist & Prasugrel & Atherothrombosis, MI & July 10, 2009 \\
\hline & Antagonist & Ticlopidine & Thrombotic stroke & July 1, 1999 \\
\hline & Antagonist & Clopidogrel & Atherosclerosis & January 1, 1900 \\
\hline \multirow{2}{*}{$\begin{array}{l}\text { Substance-P } \\
\text { receptor }\end{array}$} & Antagonist & Netupitant & \multirow[t]{2}{*}{ latrogenic emesis } & October 13, 2014 \\
\hline & Antagonist & Aprepitant & & March 26, 2003 \\
\hline \multirow[t]{2}{*}{$\begin{array}{l}\text { Estrogen receptor } \\
\text { alpha }\end{array}$} & Ligand & $\begin{array}{l}\text { Synthetic conjugated } \\
\text { estrogens, A }\end{array}$ & $\begin{array}{l}\text { Postmenopausal vulvar and vaginal atrophy, } \\
\text { vasomotor atonia }\end{array}$ & May 12, 1999 \\
\hline & Ligand & $\begin{array}{l}\text { Synthetic conjugated } \\
\text { estrogens, B }\end{array}$ & $\begin{array}{l}\text { Postmenopausal vulvar and vaginal atrophy, } \\
\text { vasomotor atonia, vaginal dryness }\end{array}$ & April 24, 2006 \\
\hline
\end{tabular}

Each row gives one "target-action" pair from the 227 "pairs" available for genomic drug repurposing, with its currently associated indications. ${ }^{\text {A4 }}$

$\mathrm{BPH}$, benign prostatic hyperplasia; BTK, Bruton's tyrosine kinase; COPD, chronic obstructive pulmonary disease; IBS, irritable bowel syndrome; MI, myocardial infarction. 


\begin{tabular}{|c|c|c|c|}
\hline \multicolumn{4}{|c|}{$\begin{array}{l}\text { Appendix Table A3. A Summary of the } 14 \text { Ongoing Repurposing Studies That Our Group Has Derived from Systematic Drug } \\
\text { Repurposability Screening Analogous to That Presented in This Publication }\end{array}$} \\
\hline Therapeutic area & Precision repurposed indication & Drug status & Developmental stage \\
\hline \multirow[t]{3}{*}{ Gastroenterology } & Recurrent Clostridium difficile colitis & Generic & Currently enrolling for phase II RCT \\
\hline & Ulcerative colitis & Reformulated generic & Preclinical/IND enabling \\
\hline & Crohn's disease & Generic & RCT planned for 2019 \\
\hline \multirow[t]{2}{*}{ Psychiatry/neurology } & Chronic fatigue from elevated NE & Generic & Biomarker study currently enrolling \\
\hline & Anti-NMDAR-associated NPSLE/lupus fog & Generic (higher dose) & Currently enrolling for phase II RCT \\
\hline Oncology & Cancer metastasis & In development & Currently enrolling for pilot RCT \\
\hline \multirow[t]{2}{*}{ Neuropathy, pain, and inflammation } & Trigeminal neuralgia & Reformulated generic & Enrolling for phase II RCT mid-2019 \\
\hline & Nerve-related headache pain & Reformulated generic & Preclinical/IND enabling \\
\hline \multirow[t]{2}{*}{ Nephrology/hematology } & Hemolytic uremic syndrome & Generic & Preclinical/IND enabling \\
\hline & Renal protection & Generic & Biomarker study planned for 2019 \\
\hline \multirow[t]{3}{*}{ Skin, fibrotic and autoimmune disease } & Wound healing in diabetic ulcer & Reformulated generic & Preclinical/IND enabling \\
\hline & Sjögren's syndrome & Generic & Enrolling for phase II RCT in late 2019 \\
\hline & Sarcoidosis & Proprietary/generic & Preclinical \\
\hline Pediatrics & Pediatric osteomyelitis & Reformulated generic & Preclinical/IND enabling \\
\hline
\end{tabular}

IND, Investigational New Drug; NE, norepinephrine; NMDAR, $N$-methyl-D-aspartate receptor; NPSLE, neuropsychiatric systemic lupus erythematosus; RCT, randomized controlled trial.

\section{APPENDIX REFERENCES}

A1. Finan C, Gaulton A, Kruger FA, et al:: The druggable genome and support for target identification and validation in drug development. Sci Trans/ Med 2017; 9:eaag 1166.

A2. Homo sapiens-Ensembl genome browser 94. https://useast.ensembl.org/Homo_ AU12sapiens/Info/Annotation (Last accessed on January 2, 2019).
A3. PROCLAIM-Misoprostol in the prevention of recurrent CDI prevent recurrence of Clostridium difficile infection with misoprostol-full text view-Clinical Trials.gov. https://clinicaltrials.gov/ct2/show/NCT03617172 (Last accessed on November 9, 2018).

A4. Documentation and Sources-DrugBank. https://www.drugbank.ca/documentation (Last accessed on April 27, 2018). 